\title{
AN ALGEBRAIC INDEPENDENCE RESULT RELATED TO A CONJECTURE OF DIXMIER ON BINARY FORM INVARIANTS
}

\author{
ABDELMALEK ABDESSELAM
}

\begin{abstract}
In order to better understand the structure of classical rings of invariants for binary forms, Dixmier proposed, as a conjectural homogeneous system of parameters, an explicit collection of invariants previously studied by Hilbert. We generalize Dixmier's collection and show that a particular subfamily is algebraically independent. Our proof relies on showing certain alternating sums of products of binomial coefficients are nonzero. Along the way we provide a very elementary proof à la Racah, namely, only using the Chu-Vandermonde Theorem, for Dixon's Summation Theorem. We also provide explicit computations of invariants, for the binary octavic, which can serve as ideal introductory examples to Gordan's 1868 method in classical invariant theory.
\end{abstract}

\section{Contents}

1. Introduction

2. Reduction to a nonvanishing statement for some combinatorial sums

3. Some combinatorial identities including those of von Szily and Dixon

4. Explicit examples

5. Why Dixmier's conjecture is not only hard but ridiculously so References

\section{INTRODUCTION}

Throughout this article we will work over the field $\mathbb{C}$ of complex numbers. For an integer $d \geq 1$, we will denote by $S_{d}$ the $(d+1)$-dimensional vector space of binary forms

$$
F(\mathbf{x})=\sum_{i=0}^{d} f_{i} x_{1}^{d-i} x_{2}^{i},
$$

namely, homogeneous polynomials of degree $d$ in the pair of variables $\mathbf{x}=\left(x_{1}, x_{2}\right)$. This space carries a natural left $S L_{2}$ action defined as follows. For a matrix

$$
g=\left(\begin{array}{ll}
g_{11} & g_{12} \\
g_{21} & g_{22}
\end{array}\right)
$$

in $S L_{2}$, we let $g \cdot \mathbf{x}=\left(g_{11} x_{1}+g_{12} x_{2}, g_{21} x_{1}+g_{22} x_{2}\right)$ and $(g \cdot F)(\mathbf{x})=F\left(g^{-1} \cdot \mathbf{x}\right)$. A polynomial $C(F, \mathbf{x})=C\left(f_{0}, \ldots, f_{d}, x_{1}, x_{2}\right)$ is called a covariant of the generic binary form of degree $d$ if it identically satisfies $C(g \cdot F, g \cdot \mathbf{x})=C(F, \mathbf{x})$ for all $g \in S L_{2}$. Such covariants form a ring $\operatorname{Cov}_{d} \subset \mathbb{C}\left[f_{0}, \ldots, f_{d}, x_{1}, x_{2}\right]$ which is bigraded by (degree, order) where "degree" refers to the degree in the $f$ variables and "order" means the degree in the $x$ variables. The order 
zero subring is the ring of invariants $\operatorname{Inv}_{d}$. The study of these rings is a classical subject in mathematics.

Minimal systems of bihomogeneous generators for $\operatorname{Cov}_{5}$ (23 generators) and $\operatorname{Cov}_{6}$ (26 generators) were obtained by Gordan in [31]. A system of generators for $\mathrm{Cov}_{8}$ was obtained by von Gall in [26, 27, 28]. He also treated the more difficult case of $\mathrm{Cov}_{7}$ in [29. Note that when a generating system of $\mathrm{Cov}_{d}$ is known, this immediately gives one for $\operatorname{Inv}_{d}$ by keeping generators of order zero. Moreover, in the classical approach of Gordan and von Gall, the study of $\operatorname{Inv}_{d}$ necessitates that of the entire ring $\operatorname{Cov}_{d}$. Progress in the study of these rings has stagnated for a long time with a few notable exceptions. Shioda rederived a minimal system for $\operatorname{Inv}_{8}$ and also found the syzygies among these generators [42. von Gall's system for $\mathrm{Cov}_{7}$ was generating but not minimal. Six elements in his list were in fact reducible. The determination of a truly minimal system of 147 generators for $\mathrm{Cov}_{7}$ has been done in [18, p. 227] and [7]. Previously, some doubt left by von Gall's work about the number of generators for $\mathrm{Inv}_{7}$ was remedied by Dixmier and Lazard [22]. Only very recently, Brouwer and Popoviciu obtained the minimal systems of generators for Inv9 (92 invariants) in [9] and for $\operatorname{Inv}_{10}$ (106 invariants) in [10]. Finally, and even more recently, in a tour de force of computational algebra Lercier and Olive [39, 36] managed to go beyond von Gall's 1888 results for covariants and determined minimal systems of generators for $\mathrm{Cov}_{9}$ (476 covariants) and $\operatorname{Cov}_{10}$ (510 covariants). These rings are notoriously difficult to present explicitly. The evident complexity displayed by the above examples is supplemented by general results of Kac [35] and Popov [40] from which one should expect high numbers of generators as well as a high homological dimension. Yet and despite this complexity, the study of rings of invariants and covariants of binary forms still is a useful task, partly because of connections to rings of Siegel modular forms (see [34] for $\operatorname{Inv}_{d}$ and the more recent [17] for $\mathrm{Cov}_{d}$ ). In an effort to find some regularity in the chaotic structure of the rings $\operatorname{Inv}_{d}$, Dixmier [21] proposed three conjectures about their Hilbert series, according to the congruence of $d \bmod 4$. In the particular case where $d$ is divisible by 4 , he conjectured an explicit homogeneous system of parameters (HSOP) with degree sequence $(2,3, \ldots, d-1)$. A recent study of such HSOP's for $\operatorname{Inv}_{d}$ can also be found in [11]. Another investigation related to Dixmier's work is [19].

For the reader's convenience, we now recall the definitions and facts from commutative algebra about HSOP's, regular sequences, etc. Our references for this material are: Brion's lectures [8, §3.4], the book by Bruns and Herzog [13, Ch. 6] and the one by Derksen and Kemper [20, $\S 2.4$ and $\S 2.5]$. Let $R=\oplus_{j \geq 0} R_{j}$ be a graded $\mathbb{C}$-algebra with $R_{0}=\mathbb{C}$, and $\theta_{1}, \ldots, \theta_{q}$ be a sequence of homogeneous elements in $R$. This sequence is called a HSOP if $\theta_{1}, \ldots, \theta_{q}$ are algebraically independent over $\mathbb{C}$ and $R$ is a finitely generated module over the subring $\mathbb{C}\left[\theta_{1}, \ldots, \theta_{q}\right]$. Assume $R$ is finitely generated as a $\mathbb{C}$-algebra, then thanks to Hilbert's homogeneous version of Noether's normalization lemma, HSOP's are guaranteed to exist. The length $q$ of such a HSOP must coincide with the Krull dimension of $R$. If $R$ is a free module over $\mathbb{C}\left[\theta_{1}, \ldots, \theta_{q}\right]$ for some HSOP $\theta_{1}, \ldots, \theta_{q}$ then $R$ is called a Cohen-Macaulay algebra. This free module property will then hold for any HSOP. Moreover, in this case a HSOP must also be a regular sequence in $R$. Recall that an $R$-regular sequence or simply a regular sequence in $R$ is a sequence of elements $\theta_{1}, \ldots, \theta_{s}$ in $R$ such that $R /\left\langle\theta_{1}, \ldots, \theta_{s}\right\rangle \neq 0$ and for all $i=1, \ldots, s$, multiplication by $\theta_{i}$ in $R /\left\langle\theta_{1}, \ldots, \theta_{i-1}\right\rangle$ is injective. For $R=\operatorname{Inv}_{d}$, $q=\operatorname{dim}\left(S_{d}\right)-\operatorname{dim}\left(S L_{2}\right)=(d+1)-3=d-2$, since the stabilizer of a generic $F \in S_{d}$ is finite. By the Hochster-Roberts Theorem, $R=\operatorname{Inv}_{d}$ is Cohen-Macaulay. Moreover, a regular 
sequence $\theta_{1}, \ldots, \theta_{s}$ in $\operatorname{Inv}_{d}$ must have $s \leq q=d-2$ and can always be completed into a HSOP. One should also mention a criterion due to Hilbert which characterizes sequences of homogeneous elements $\theta_{1}, \ldots, \theta_{d-2}$ that are HSOP's. Namely, to be an HSOP, a necessary and sufficient condition is that $\theta_{1}, \ldots, \theta_{d-2}$ provide set-theoretic equations for the nullcone $\mathscr{N}_{d}=\left\{F \in S_{d} \mid \forall J \in \operatorname{Inv}_{d}, J(F)=0\right\}$. Finally, since this notion will play a role later in this article, let us call two sequences of homogeneous elements $\theta_{1}, \ldots, \theta_{s}$ and $\theta_{1}^{\prime}, \ldots, \theta_{s}^{\prime}$ triangularly related if for all $i$ one can write $\theta_{i}^{\prime}=\gamma_{i} \theta_{i}+P_{i}\left(\theta_{1}, \ldots, \theta_{i-1}\right)$ for some nonzero scalars $\gamma_{i} \in \mathbb{C}$ and for some polynomials $P_{i}$ with complex coefficients. Clearly, this is an equivalence relation which preserves the HSOP and regular sequence properties.

Recall that for $0 \leq k \leq \min (m, n)$ and for $F \in S_{m}$ and $G \in S_{n}$, one has the classical notion of transvectant $(F, G)_{k} \in S_{m+n-2 k}$ given by

$$
(F, G)_{k}=\frac{(m-k) !(n-k) !}{m ! n !} \sum_{l=0}^{k}(-1)^{l}\left(\begin{array}{c}
k \\
l
\end{array}\right) \frac{\partial^{k} F}{\partial x_{1}^{k-l} \partial x_{2}^{l}} \frac{\partial^{k} G}{\partial x_{1}^{l} \partial x_{2}^{k-l}} .
$$

This bilinear operation realizes the $S L_{2}$-equivariant projection $S_{m} \otimes S_{n} \rightarrow S_{m+n-2 k}$ of the Clebsch-Gordan decomposition. We now assume $d=2 k$ with $k$ even and take $n \geq k$. For fixed $F \in S_{d}$, consider the linear map

$$
\begin{aligned}
\mathcal{L}_{n}^{F}: S_{n} & \longrightarrow S_{n} \\
G & \longmapsto(F, G)_{k}
\end{aligned}
$$

and for $1 \leq p \leq n+1$ we denote by $\mathscr{H}_{n, p}(F)$ the coefficient of $\lambda^{p}$ in the characteristic polynomial $\operatorname{det}\left(\lambda \mathrm{Id}-\mathcal{L}_{n}^{F}\right)$ of the map $\mathcal{L}_{n}^{F}$. These polynomials in $F$ were introduced by Hilbert in his Königsberg Habilitationsschrift [33] where he showed they are $S L_{2}$ invariants of $F$ (see also [37, §3]).

Conjecture 1. (Dixmier [21, Conjecture 3'])

When $d$ is divisible by $4, \mathscr{H}_{d-2,2}(F), \mathscr{H}_{d-2,3}(F), \ldots, \mathscr{H}_{d-2, d-1}(F)$ form a HSOP for $\operatorname{Inv}_{d}$.

An equivalent reformulation of Dixmier's Conjecture is that, for $d$ divisible by 4 ,

$$
\mathscr{P}_{d-2,2}(F), \mathscr{P}_{d-2,3}(F), \ldots, \mathscr{P}_{d-2, d-1}(F)
$$

form a HSOP for $\operatorname{Inv}_{d}$, where

$$
\mathscr{P}_{n, p}(F)=\operatorname{tr}\left[\left(\mathcal{L}_{n}^{F}\right)^{p}\right] .
$$

Indeed, $\mathscr{H}_{d-2,2}(F), \mathscr{H}_{d-2,3}(F), \ldots, \mathscr{H}_{d-2, d-1}(F)$ and $\mathscr{P}_{d-2,2}(F), \mathscr{P}_{d-2,3}(F), \ldots, \mathscr{P}_{d-2, d-1}(F)$ are triangularly related. This follows from the classical explicit formulas relating the elementary symmetric functions $\pm \mathscr{H}_{n, p}$ of the eigenvalues of $\mathcal{L}_{n}^{F}$ to the power sums $\mathscr{P}_{n, p}$. Note that one has identically $\mathscr{H}_{n, 1}=\mathscr{P}_{n, 1}=0$ since binary forms have no linear invariant. As mentioned above, a HSOP for $\operatorname{Inv}_{d}$ must have exactly $d-2$ elements. This explains why Dixmier picked the sequence corresponding to $n=d-2$ for his conjecture. We propose to enlarge Dixmier conjecture as follows.

Conjecture 2. For $d=2 k$ with $k$ even and for $k \leq n \leq 2 k-2, \mathscr{P}_{n, 2}, \mathscr{P}_{n, 3}, \ldots, \mathscr{P}_{n, n+1}$ is a regular sequence in the ring $\operatorname{Inv}_{d}$.

As will be made clear in $§ 5$, Dixmier's Conjecture is very difficult. Our Conjecture 2 suggests a more progressive approach of establishing the regular sequence property for increasing values of $n$. The main result of this article is the following modest step in this direction. 
Theorem 1. With the same hypotheses and notation as in Conjecture Q , we have that $\mathscr{P}_{k, 2}, \mathscr{P}_{k, 3}, \ldots, \mathscr{P}_{k, k+1}$ are algebraically independent.

The main body of the proof of this theorem is in $\S 2$ where we compute the Jacobian matrix of the invariants at a suitable point or binary form $\mathbb{F}$. Algebraic independence follows from this matrix having full rank which amounts to showing the nonvanishing of some combinatorial sums $\Upsilon_{m}$. This nonvanishing is established in $\S 3$ by revisiting recent work of Guo, Jouhet and Zeng [32]. In fact, one can write $\Upsilon_{m}$ as an explicit sum of positive terms [32, theorem 1.2] which itself is a consequence of a rather intimidating $q$-hypergeometric multisum identity of Andrews [6, Theorem 4]. In $\S 3$, we follow a more elementary approach via a recursive formula [32, Lemma 2.1]. This gives us an opportunity to introduce several improvements on the derivation in 32]: we do not use the Pfaff-Saalschütz identity but only the simpler Chu-Vandermonde Theorem. We start the recursion at $m=2$ instead of $m=3$ which forced the authors of [32] to invoke rather than deduce Dixon's Theorem. As a pleasant surprise, we obtained a proof of the latter which is very elementary and perhaps new. Note that the computations in $\S 2$ were originally done using the graphical calculus developed in [1, $\S 2]$. The derivatives with respect to $f_{s}, 0 \leq s \leq d$, of an invariant $\mathscr{P}$ are best packaged into its first evectant (see [16, §5] for a definition). We then wrote down a graphical formula for the covariant $\mathscr{W}$ obtained as the homogeneous Wronskian of these evectants (see 2] for a recent study of such Wronskians from an invariant-theoretic perspective). The existence of a point where the Jacobian matrix has full rank is equivalent to the covariant $\mathscr{W}$ not being identically zero. With the help of our graphical representation for $\mathscr{W}$, and also with some inspiration taken from [37, §3], we found a suitable point of specialization $\mathbb{F}$ in the nullcone. However, in order to make our proof accessible to a wider audience, we erased our footsteps in our writing of $\S 2$ which can be read without knowledge of [1, §2] and which only requires elementary linear algebra and multivariable calculus. In $\S 4$, we explicitly compute the invariants $\mathscr{P}$ that are relevant for Theorem 11, in the case of the binary quartic (trivial) and that of the binary octavic (involved but instructive). In $\S 5$, which assumes some familiarity with the graphical calculus of [1, §2], we provide a hopefully insightful discussion of Dixmier's Conjecture by extracting some of the combinatorial difficulties it contains.

\section{ReduCtion to A NONVANishing STATEMENT FOR SOME COMBINATORIAL SUmS}

Throughout this article, we will, similarly to Iverson's bracket, use the notation $\mathbb{1}\{\cdots\}$ for the indicator function of the condition between braces. Let $\mathcal{B}$ denote the basis of monomials

$x_{1}^{k-i} x_{2}^{i}, 0 \leq i \leq k$ for the space $S_{k}$. For a linear operator $M: S_{k} \rightarrow S_{k}$ we will denote by $[M]_{i j}$ the matrix elements of this operator in the basis $\mathcal{B}$. Let $\mathscr{J}(F)=\left(\mathscr{J}_{r, s}\right)_{\substack{2 \leq r \leq k+1 \\ 0 \leq s \leq 2 k}}$ denote the Jacobian matrix of the invariants $\mathscr{P}_{k, 2}, \ldots, \mathscr{P}_{k, k+1}$ at some binary form $F \in S_{d}$. Namely,

$$
\mathscr{J}_{r, s}=\frac{\partial}{\partial f_{s}} \mathscr{P}_{k, r}(F)
$$

For $0 \leq s \leq d=2 k$ and $r \geq 1$, by the multivariate chain rule and the cyclic property of the trace we have

$$
\frac{\partial}{\partial f_{s}} \mathscr{P}_{k, r}(F)=r \times \sum_{\substack{i, j=0 \\ 4}}^{k}\left[\left(\mathcal{L}_{k}^{F}\right)^{r-1}\right]_{i j} \frac{\partial}{\partial f_{s}}\left[\mathcal{L}_{k}^{F}\right]_{j i}
$$


A straightforward computation using (1) gives, in general for $0 \leq i \leq m, 0 \leq j \leq n$ and $0 \leq k \leq \min (m, n)$

$$
\left(x_{1}^{m-i} x_{2}^{i}, x_{1}^{n-j} x_{2}^{j}\right)_{k}=T_{i, j}^{m, n, k} x_{1}^{m+n-k-i-j} x_{2}^{i+j-k}
$$

where

$$
\begin{aligned}
T_{i, j}^{m, n, k}= & \frac{(m-k) !(n-k) ! k ! i !(m-i) ! j !(n-j) !}{m ! n !} \\
& \times \sum_{l=\max (0, k-j, k-m+i)}^{\min (i, n-j, k)} \frac{(-1)^{l}}{l !(k-l) !(i-l) !(n-j-l) !(j-k+l) !(m-i-k+l) !} .
\end{aligned}
$$

In the application of this formula to our case of interest, the sum reduces to a single term and therefore, when $0 \leq i, j \leq k$,

$$
\left[\mathcal{L}_{k}^{F}\right]_{i j}=f_{i-j+k} \times T_{i-j+k, j}^{2 k, k, k}
$$

with

$$
T_{i-j+k, j}^{2 k, k, k}=(-1)^{j} \times \frac{k !(k-i+j) !(k+i-j) !}{(2 k) ! i !(k-i) !}
$$

since $k$ is even. We now specialize our calculation of the Jacobian matrix to the particular unstable form $\mathbb{F}=x_{1}^{k-1} x_{2}^{k+1}$ which has as coefficients $f_{s}=\mathbb{1}\{s=k+1\}$, for $0 \leq s \leq 2 k$. Thus

$$
\left[\mathcal{L}_{k}^{\mathbb{F}}\right]_{i j}=\mathbb{1}\{i=j+1\} \times \frac{(-1)^{j}\left(\begin{array}{c}
k \\
j+1
\end{array}\right)}{\left(\begin{array}{c}
2 k \\
k+1
\end{array}\right)}
$$

and the corresponding matrix essentially is a nilpotent Jordan-like matrix with nonzero entries only immediately below the diagonal. One also has

$$
\left.\frac{\partial}{\partial f_{s}}\left[\mathcal{L}_{k}^{F}\right]_{j i}\right|_{F=\mathbb{F}}=\mathbb{1}\{s=j-i+k\} \times T_{j-i+k, i}^{2 k, k, k} .
$$

Let $\mathbb{J}=\left(\mathbb{J}_{r, s}\right)_{\substack{2 \leq r \leq k+1 \\ 0 \leq s \leq 2 k}}$ denote the Jacobian matrix $\mathscr{J}(F)$ specialized at $F=\mathbb{F}$. Then an immediate computation using (2) gives

$$
\mathbb{J}_{r, s}=\frac{r \times(-1)^{\left(\begin{array}{c}
r \\
2
\end{array}\right) \times 11\{s=k-r+1\}}}{\left(\begin{array}{c}
2 k \\
k+r-1
\end{array}\right)\left(\begin{array}{c}
2 k \\
k+1
\end{array}\right)^{r-1}} \times \mathscr{N}_{k, r}
$$

where

$$
\mathscr{N}_{k, r}=\sum_{j=0}^{k-r+1}(-1)^{r j}\left(\begin{array}{c}
k \\
j
\end{array}\right)\left(\begin{array}{c}
k \\
j+1
\end{array}\right) \cdots\left(\begin{array}{c}
k \\
j+r-1
\end{array}\right) .
$$

Consider the maximal $k \times k$ minor

$$
\widehat{\mathbb{J}}=\operatorname{det}\left[\left(\mathbb{J}_{r, s}\right)_{\substack{2 \leq r \leq k+1 \\ 0 \leq s \leq k-1}}\right] .
$$

Then $\widehat{\mathbb{J}}$ is equal to an obviously nonzero number times the product $\prod_{r=2}^{k+1} \mathscr{N}_{k, r}$.

The algebraic independence in Theorem 1 follows from $\widehat{\mathbb{J}} \neq 0$ which itself reduces to showing that for all $r, 2 \leq r \leq k+1$, we have $\mathscr{N}_{k, r} \neq 0$. The case where $r$ is even is of course trivial, whereas the more involved alternating sum situation where $r$ is odd will be taken care of in Proposition 7 below. 


\section{Some Combinatorial identities including those of von Szily And Dixon}

In this section we will adopt the following convention regarding ratios of products of factorials. The $a$ 's and $b$ 's being elements of $\mathbb{Z}$, we let

$$
\frac{a_{1} ! \cdots a_{m} !}{b_{1} ! \cdots b_{n} !}:=a_{1} ! \times \cdots \times a_{m} ! \times \frac{1}{b_{1} !} \times \cdots \times \frac{1}{b_{n} !}
$$

where, for any $n \in \mathbb{Z}$, we set by definition

$$
n !=\left\{\begin{array}{cl}
\operatorname{ordinary} n ! & \text { if } n \geq 0, \\
0 & \text { if } n<0,
\end{array}\right.
$$

and

$$
\frac{1}{n !}=\left\{\begin{array}{cl}
\operatorname{ordinary} \frac{1}{n !} & \text { if } n \geq 0 \\
0 & \text { if } n<0
\end{array}\right.
$$

Beware that with such a convention $\frac{n !}{n !}$ is not equal to 1 but rather $\mathbb{1}\{n \geq 0\}$. For any $n, k \in \mathbb{Z}$, we also define the binomial coefficients $\left(\begin{array}{l}n \\ k\end{array}\right)=\frac{n !}{k !(n-k) !}$ with the previous convention enforced. In particular, the coefficients are zero unless $0 \leq k \leq n$. In all the following combinatorial sums, the range of summation will be $\mathbb{Z}$ and therefore omitted.

Proposition 1. (Chu-Vandermonde) $\forall a, b, c \in \mathbb{Z}$,

$$
\sum_{k}\left(\begin{array}{l}
a \\
k
\end{array}\right)\left(\begin{array}{c}
b \\
c-k
\end{array}\right)=\mathbb{1}\left\{\begin{array}{l}
a \geq 0 \\
b \geq 0
\end{array}\right\}\left(\begin{array}{c}
a+b \\
c
\end{array}\right) .
$$

Proof: Both sides of the equation vanish unless $a \geq 0$ and $b \geq 0$ which we now assume. Taking the sum of the inequalities $k \geq 0$ and $c-k \geq 0$ implies $c \geq 0$. So when $c<0$ both sides vanish. Taking the sum of the inequalities $k \leq a$ and $c-k \leq b$ implies $c \leq a+b$. So also when $c>a+b$ both sides vanish. The remaining case is when $0 \leq c \leq a+b$. Take $A$ a set of cardinality $a$. Take $B$ a set of cardinality $b$ that is disjoint from $A$. Finally, count subsets $C \subset A \cup B$ with cardinality $c$ by conditioning on the cardinality $k$ of $C \cap A$.

For our purposes a more practical form of the Chu-Vandermonde convolution identity is the following.

Corollary 1. $\forall a, b, c \in \mathbb{Z}$,

$$
\left(\begin{array}{c}
2 a \\
a+c
\end{array}\right)\left(\begin{array}{c}
2 b \\
b+c
\end{array}\right)=\frac{(2 a) !(2 b) !}{(a+b) !} \sum_{l} \frac{1}{(a-l) !(b-l) !(l+c) !(l-c) !} .
$$

Proof: The summand in the RHS includes the indicator functions of $a-l \geq 0$ and $l-c \geq 0$ and therefore the sum of these inequalities gives $a-c \geq 0$. Likewise, the conditions $b-l \geq 0$ and $c+l \geq 0$ are included and so is their consequence $b+c \geq 0$. We can thus multiply and divide by $(a-c)$ ! and $(b+c)$ ! which gives

$$
\operatorname{RHS}=\frac{(2 a) !(2 b) !}{(a+b) !(a-c) !(b+c) !} \times \sum_{l}\left(\begin{array}{c}
a-c \\
l-c
\end{array}\right)\left(\begin{array}{c}
b+c \\
b-l
\end{array}\right) .
$$

Changing variables to $k=l-c$ and applying Proposition 1 gives

$$
\operatorname{RHS}=\frac{(2 a) !(2 b) !}{(a+b) !(a-c) !(b+c) !} \times \mathbb{1}\left\{\begin{array}{c}
a-c \geq 0 \\
b+c \geq 0
\end{array}\right\}\left(\begin{array}{c}
a+b \\
b-c
\end{array}\right)
$$


which reduces to LHS after cleaning up the expression, taking our convention into account.

For all $m \geq 1$ and $a_{1}, \ldots, a_{m} \in \mathbb{Z}$, let

$$
\Upsilon_{m}\left(a_{1}, \ldots, a_{m}\right)=\sum_{k}(-1)^{k}\left(\begin{array}{c}
2 a_{1} \\
a_{1}+k
\end{array}\right)\left(\begin{array}{c}
2 a_{2} \\
a_{2}+k
\end{array}\right) \cdots\left(\begin{array}{c}
2 a_{m} \\
a_{m}+k
\end{array}\right) .
$$

These alternating combinatorial sums satisfy the following recursion due to Guo, Jouhet and Zheng [32, Lemma 2.1].

Proposition 2. $\forall m \geq 2, \forall a_{1}, \ldots, a_{m} \in \mathbb{Z}$,

$$
\Upsilon_{m}\left(a_{1}, \ldots, a_{m}\right)=\sum_{l} \frac{\left(2 a_{1}\right) !\left(2 a_{2}\right) !}{\left(a_{1}+a_{2}\right) !(2 l) !\left(a_{1}-l\right) !\left(a_{2}-l\right) !} \times \Upsilon_{m-1}\left(l, a_{3}, \ldots, a_{m}\right) .
$$

Proof: Using $Q$ as a placeholder for the product $\prod_{2<i \leq m}\left(\begin{array}{c}2 a_{i} \\ a_{i}+k\end{array}\right)$, we have

$$
\Upsilon_{m}\left(a_{1}, \ldots, a_{m}\right)=\sum_{k}(-1)^{k}\left(\begin{array}{c}
2 a_{1} \\
a_{1}+k
\end{array}\right)\left(\begin{array}{c}
2 a_{2} \\
a_{2}+k
\end{array}\right) \times Q
$$

in which we insert the identity of Corollary 1 for the first two binomial coefficients with the result

$$
\Upsilon_{m}\left(a_{1}, \ldots, a_{m}\right)=\sum_{k} \sum_{l} \frac{(-1)^{k}\left(2 a_{1}\right) !\left(2 a_{2}\right) !}{\left(a_{1}+a_{2}\right) !\left(a_{1}-l\right) !\left(a_{2}-l\right) !(l-k) !(l+k) !} \times Q .
$$

The summand includes the indicator function of the conditions $l-k \geq 0$ and $l+k \geq 0$ which imply $2 l \geq 0$. It is thus legitimate to multiply and divide by $(2 l)$ !. Also note that only finitely many pairs $(k, l)$ contribute to the last sum because of the implied conditions $0 \leq l \leq \min \left(a_{1}, a_{2}\right)$ and $-l \leq k \leq l$. Hence Fubini's Theorem applies and one can write

$$
\Upsilon_{m}\left(a_{1}, \ldots, a_{m}\right)=\sum_{l} \sum_{k} \frac{(-1)^{k}\left(2 a_{1}\right) !\left(2 a_{2}\right) !}{\left(a_{1}+a_{2}\right) !\left(a_{1}-l\right) !\left(a_{2}-l\right) !(2 l) !} \times\left(\begin{array}{c}
2 l \\
l+k
\end{array}\right) \times Q .
$$

The result then follows from the definition of $\Upsilon_{m-1}\left(l, a_{3}, \ldots, a_{m}\right)$.

We now look at the first three simplest cases.

Proposition 3. $\forall a_{1} \in \mathbb{Z}$, we have

$$
\Upsilon_{1}\left(a_{1}\right)=\mathbb{1}\left\{a_{1}=0\right\} .
$$

Proof: By changing variables to $j=a_{1}+k$, we have from the definition

$$
\Upsilon_{1}\left(a_{1}\right)=(-1)^{a_{1}} \sum_{j}(-1)^{j}\left(\begin{array}{c}
2 a_{1} \\
j
\end{array}\right) \text {. }
$$

If $a_{1}<0$, the RHS is zero by convention. If $a_{1}=0$, the RHS reduces to $\left(\begin{array}{l}0 \\ 0\end{array}\right)=1$. If $a_{1}>0$, Newton's Binomial Theorem gives RHS $=(-1)^{a_{1}}(1-1)^{a_{1}}=0$.

The case $m=2$ is already more interesting since it amounts to the von Szily identity [44] for super-Catalan numbers (see, e.g., 30]). 
Proposition 4. (von Szily) $\forall a_{1}, a_{2} \in \mathbb{Z}$,

$$
\Upsilon_{2}\left(a_{1}, a_{2}\right)=\frac{\left(2 a_{1}\right) !\left(2 a_{2}\right) !}{\left(a_{1}+a_{2}\right) ! a_{1} ! a_{2} !} .
$$

Proof: Insert the identity (5) inside (4) and the result follows immediately.

The case $m=3$ is Dixon's Summation Theorem for terminating ${ }_{3} F_{2}$ hypergeometric series.

Proposition 5. (Dixon) $\forall a_{1}, a_{2}, a_{3} \in \mathbb{Z}$,

$$
\Upsilon_{3}\left(a_{1}, a_{2}, a_{3}\right)=\frac{\left(2 a_{1}\right) !\left(2 a_{2}\right) !\left(2 a_{3}\right) !\left(a_{1}+a_{2}+a_{3}\right) !}{\left(a_{1}+a_{2}\right) !\left(a_{1}+a_{3}\right) !\left(a_{2}+a_{3}\right) ! a_{1} ! a_{2} ! a_{3} !} .
$$

Proof: We insert (6) inside (41) and get

$$
\Upsilon_{3}\left(a_{1}, a_{2}, a_{3}\right)=\sum_{l} \frac{\left(2 a_{1}\right) !\left(2 a_{2}\right) !}{\left(a_{1}+a_{2}\right) !(2 l) !\left(a_{1}-l\right) !\left(a_{2}-l\right) !} \times \frac{(2 l) !\left(2 a_{3}\right) !}{\left(l+a_{3}\right) ! l ! a_{3} !} .
$$

Since the factor $\frac{1}{l !}$ includes $\mathbb{1}\{l \geq 0\}$ one may cancel (2l)! above and below. Similarly the factors $\left(2 a_{2}\right)$ ! and $\left(2 a_{3}\right)$ ! provide the condition $a_{2}+a_{3} \geq 0$ which allows us to multiply and divide by $\left(a_{2}+a_{3}\right)$ !. After regrouping we obtain

$$
\Upsilon_{3}\left(a_{1}, a_{2}, a_{3}\right)=\frac{\left(2 a_{1}\right) !\left(2 a_{2}\right) !\left(2 a_{3}\right) !}{\left(a_{1}+a_{2}\right) ! a_{3} ! a_{1} !\left(a_{2}+a_{3}\right) !} \times \sum_{l}\left(\begin{array}{c}
a_{1} \\
l
\end{array}\right)\left(\begin{array}{c}
a_{2}+a_{3} \\
a_{2}-l
\end{array}\right) .
$$

Finally, summing over $l$ using Proposition 1 and cleaning up the resulting expression gives the desired identity.

Remark 1. The particular case $a_{1}=a_{2}=a_{3}$ of Dixon's Theorem was conjectured by Morley and proved in 23]. The general case is also due to Dixon 24] although it is sometimes attributed to Fjeldstad [25]. It has also been proved by Racah [41, Appendix A]. There are of course many proofs available, but to the best of our knowledge this is perhaps the first proof which only uses the Chu-Vandermonde identity in both directions, in the spirit of Racah's derivation of his single sum formula for $6 j$ symbols [41, Appendix B].

For $m>3$ there are no more simple closed formulas for $\Upsilon_{m}\left(a_{1}, \ldots, a_{m}\right)$, since, even when $a_{1}=\cdots=a_{m}$, no such formula exists as shown by de Bruijn [12, §4.7]. Nevertheless the following result will be enough to prove our main theorem.

Proposition 6. $\forall m \geq 2, \forall a_{1}, \ldots, a_{m} \geq 0$, we have $\Upsilon_{m}\left(a_{1}, \ldots, a_{m}\right)>0$.

Proof: For $m=2$ this follows from the explicit formula in (6). The general case follows by a simple induction on $m$. Namely, for $m \geq 3$, apply (4) and bound the sum from below by the single term with $l=0$. This gives

$$
\Upsilon_{m}\left(a_{1}, \ldots, a_{m}\right) \geq \frac{\left(2 a_{1}\right) !\left(2 a_{2}\right) !}{\left(a_{1}+a_{2}\right) a_{1} ! a_{2} !} \times \Upsilon_{m-1}\left(0, a_{3}, \ldots, a_{m}\right)>0 .
$$

We now relate the combinatorial sums of this section to the numerical quantities $\mathscr{N}_{k, r}$ from $\S 2$.

Proposition 7. For all integers $p, q$ with $1 \leq q \leq p$,

$$
\mathscr{N}_{2 p, 2 q+1}=\frac{(-1)^{p+q}(2 p) !^{2 q+1}}{\prod_{\nu=0}^{2 q}[2(p-q+\nu)] !} \times \Upsilon_{8}{ }_{2 q+1}(p-q, p-q+1, \ldots, p+q+1) .
$$


Proof: In the formula (3) for $\mathscr{N}_{k, r}$ with $k=2 p$ and $r=2 q+1$ and for $0 \leq j \leq k-r+1$, we write

$$
\left(\begin{array}{c}
k \\
j
\end{array}\right)\left(\begin{array}{c}
k \\
j+1
\end{array}\right) \cdots\left(\begin{array}{c}
k \\
j+r-1
\end{array}\right)=\frac{k !^{r}}{D}
$$

where the denominator is

$$
\begin{aligned}
D & =\prod_{\nu=0}^{r-1}[(j+\nu) !(k-j-\nu) !] \\
& =\prod_{\nu=0}^{r-1}(j+\nu) ! \times \prod_{\nu=0}^{r-1}(k-j-\nu) ! \\
& =\prod_{\nu=0}^{r-1}(j+\nu) ! \times \prod_{\nu=0}^{r-1}(k-j-r+1+\nu) !
\end{aligned}
$$

where in the last product we reversed the order of factors, i.e., changed $\nu$ to $r-1-\nu$. As a result,

$$
\begin{aligned}
\frac{k !^{r}}{D} & =\frac{k !^{r}}{\prod_{\nu=0}^{r-1}[(j+\nu) !(k-j-r+1+\nu) !]} \\
& =\frac{k !^{r}}{\prod_{\nu=0}^{r-1}(k-r+1+2 \nu) !} \times \prod_{\nu=0}^{r-1}\left(\begin{array}{c}
k-r+1+2 \nu \\
j+\nu
\end{array}\right) .
\end{aligned}
$$

We then change the summation index from $j$ to $i=j+q-p$, write $k, r$ in terms of $p, q$, use the definition of $\Upsilon_{m}$ and clean up the final expression to get the desired identity.

The proof of Theorem 1 is now complete.

\section{EXPLICIT EXAMPLES}

In this section we provide explicit computations for some of our invariants $\mathscr{P}$. We will assume familiarity with the classical symbolic method and notation, as explained in [1, $\S 2]$. A superficial glance at the following calculations may give the impression that they are mindless and straightforward. They are not! They in fact provide a rare opportunity for the reader to get "hands on" experience with Gordan's 1868 reduction algorithm [31]. The latter uses as a guide the graph-theoretic notion of $p$-edge-connectivity. One of the main ideas is to use variants of the Grassmann-Plücker relation in order to create pairs of vertices with a high number of edges joining them. This means creating factors $(a b)^{p}$ with $p>\frac{d}{2}$. The highest such exponent in a graph or symbolic bracket monomial is also called the "grade" (see [46] for a modern perspective). When present, such factors $(a b)^{p}$ result in the vertices $a$ and $b$ having less than $\frac{d}{2}$ remaining connections to the rest of the graph. Applying the Clebsch-Gordan identity between these remaining edges emanating from $a$ and $b$ only produces transvections $(\cdot, \cdot)_{q}$ with $q<\frac{d}{2}$ which is important for the success of the reduction process. The most technically delicate part of Gordan's algorithm is the special treatment of forms of degree divisible by 4 which have a degree two covariant of the same order as the form itself, namely $(a b)^{\frac{d}{2}} a_{x}^{\frac{d}{2}} b_{x}^{\frac{d}{2}}$. In order to eliminate the troublesome factors $(a b)^{\frac{d}{2}}$, Gordan had to consider covariants of degree three and he discovered clever identities [31, §7] which allowed him to improve the grade from $\frac{d}{2}$ to some higher exponent. Our invariants 
$\mathscr{P}_{k, n}$ featuring in Theorem 1 are entirely made of factors $(a b)^{\frac{d}{2}}$ and thus take us to the very technical heart of Gordan's method. We do not exactly use his identities but resort to gradeimproving identities that are similar in spirit. The most important one is Eq. (8) below. Finally, note that the following computations were first done graphically and then converted to the more compact classical symbolic notation. The reader is strongly encouraged to follow the calculations by drawing directed graphs "on the side" where symbolic letters $a$, $b$, etc. correspond to vertices and brackets $(a b),(a c)$, etc. correspond to directed edges.

In general, when $d=2 k$ with $k$ even and for $p=2, \ldots, k+1$, we have the "cyclic" symbolic formula

$$
\mathscr{P}_{k, p}(F)=\left(a^{(1)} a^{(2)}\right)^{k}\left(a^{(2)} a^{(3)}\right)^{k}\left(a^{(3)} a^{(4)}\right)^{k} \cdots\left(a^{(p-1)} a^{(p)}\right)^{k}\left(a^{(p)} a^{(1)}\right)^{k}
$$

where $a^{(1)}, \ldots, a^{(p)}$ are symbolic letters for the form $F$. The particular case of the binary quartic is rather trivial, since we get:

$$
\mathscr{P}_{2,2}=(a b)^{2}(b a)^{2}=(F, F)_{4} \quad, \quad \mathscr{P}_{2,3}=(a b)^{2}(b c)^{2}(c a)^{2}=\left(F,(F, F)_{2}\right)_{4} .
$$

It has been known since the middle of the 19th century that these two invariants generate $\mathrm{Inv}_{4}$ and are algebraically independent. Thus the HSOP and regular sequence properties hold trivially. Note that the vanishing of $\mathscr{P}_{2,2}$ detects equianharmonic configurations of four points on $\mathbb{P}^{1}(\mathbb{C})$, while that of the catalecticant $\mathscr{P}_{2,3}$ detects binary quartics which are limits of sums of two fourth powers of linear forms.

Let us now focus on the more challenging case $d=8$ and explicitly compute the invariants $\mathscr{P}_{4,2}, \mathscr{P}_{4,3}, \mathscr{P}_{4,4}, \mathscr{P}_{4,5}$ relevant to our Theorem 1, in terms of the invariants listed by Shioda [42. In the latter article, Shioda proved that $\operatorname{Inv}_{8}$ is generated by elements $J_{2}, J_{3}, \ldots, J_{10}$ of respective degree $2,3, \ldots, 10$. He showed $J_{2}, \ldots, J_{7}$ are algebraically independent and also proved that $\operatorname{Inv}_{8}$ is a free module over $\mathbb{C}\left[J_{2}, \ldots, J_{7}\right]$ with basis given by $1, J_{8}, J_{9}, J_{10}, J_{9}^{2}$. Namely, he explicitly proved the Cohen-Macaulay property for Inv 8 . From his list, we will need

$$
\begin{aligned}
& J_{2}=(F, F)_{8}=(a b)^{8} \\
& J_{3}=\left(F,(F, F)_{4}\right)_{8}=(a b)^{4}(a c)^{4}(b c)^{4} \\
& J_{4}=\left((F, F)_{6},(F, F)_{6}\right)_{4} \\
& J_{5}=\left(\left(F,(F, F)_{6}\right)_{4},(F, F)_{6}\right)_{4}=(a b)^{6}(a c)^{2}(b c)^{2}(c d)^{2}(c e)^{2}(d e)^{6}
\end{aligned}
$$

These same invariants are respectively denoted by $A, B, C, f_{k, k}$ in [26]. We clearly have $\mathscr{P}_{4,2}=J_{2}$ and $\mathscr{P}_{4,3}=J_{3}$. However, the computation of $\mathscr{P}_{4,4}$ and $\mathscr{P}_{4,5}$ is more involved and necessitates a detailed study of degree three covariants of order four and eight.

For $\lambda=\left(\lambda_{1}, \lambda_{2}, \lambda_{3}\right)$, with $\lambda_{1} \geq \lambda_{2} \geq \lambda_{3} \geq 0$, an integer partition of 4 , we define

$$
A_{\lambda}=(a b)^{\lambda_{3}+2}(a c)^{\lambda_{2}+2}(b c)^{\lambda_{1}+2} a_{x}^{\lambda_{1}} b_{x}^{\lambda_{2}} c_{x}^{\lambda_{3}}
$$

It is easy to that the $A_{\lambda}$ linearly generate the space of covariants of degree 3 and order 4 . Note that we have the basic identity

$$
a_{x}(b c)=b_{x}(a c)-c_{x}(a b)
$$

which allows one to rewrite any of the above three terms using the other two. Consider $A_{31}=(a b)^{2}(a c)^{3}(b c)^{5} a_{x}^{3} b_{x}$ and use the basic identity to rewrite $b_{x}(a c)$. We then get

$$
A_{31}=(a b)^{2}(a c)^{2}(b c)^{6} a_{x}^{4}+(a b)^{3}(a c)^{2}(b c)^{5} a_{x}^{3} c_{x} .
$$


Exchanging the symbolic letters $b$ and $c$ in the second term and using the antisymmetry of brackets $(c b)=-(b c)$, one obtains the relation $A_{31}=A_{4}-A_{31}$ and thus $A_{31}=\frac{1}{2} A_{4}$. Rewriting $a_{x}(b c)$ inside $A_{211}=(a b)^{3}(a c)^{3}(b c)^{4} a_{x}^{2} b_{x} c_{x}$ gives the relation $A_{211}=-2 A_{211}$ and thus $A_{211}=0$. Finally, rewriting $a_{x}(b c)$ inside $A_{22}=(a b)^{2}(a c)^{4}(b c)^{4} a_{x}^{2} b_{x}^{2}$ gives

$$
A_{22}=A_{31}+A_{211}=\frac{1}{2} A_{4}=\frac{1}{2}\left(F,(F, F)_{6}\right)_{4} .
$$

We now consider partitions $\lambda=\left(\lambda_{1}, \lambda_{2}, \lambda_{3}\right)$ of 8 and define

$$
B_{\lambda}=(a b)^{\lambda_{3}}(a c)^{\lambda_{2}}(b c)^{\lambda_{1}} a_{x}^{\lambda_{1}} b_{x}^{\lambda_{2}} c_{x}^{\lambda_{3}} .
$$

These bracket monomials linearly generate the space of of covariants of degree 3 and order 8. Rewriting $c_{x}(a b)$ inside $B_{521}=(a b)(a c)^{2}(b c)^{5} a_{x}^{5} b_{x}^{2} c_{x}$ gives $B_{521}=B_{53}-B_{62}$. Rewriting $a_{x}(b c)$ inside $B_{422}=(a b)^{2}(a c)^{2}(b c)^{4} a_{x}^{4} b_{x}^{2} c_{x}^{2}$ gives $B_{422}=2 B_{332}$. Rewriting $a_{x}(b c)$ inside $B_{431}=$ $(a b)(a c)^{3}(b c)^{4} a_{x}^{4} b_{x}^{3} c_{x}$ gives $B_{431}=-B_{431}-B_{332}$ and thus $B_{332}=-2 B_{431}$. However, one can also rewrite $b_{x}(a c)$ inside $B_{431}$ again which results in the relation

$$
B_{431}=B_{521}+B_{422}=B_{521}-4 B_{431}
$$

and therefore

$$
B_{431}=\frac{1}{5} B_{521}=\frac{1}{5} B_{53}-\frac{1}{5} B_{62} .
$$

Finally, rewriting $a_{x}(b c)$ inside $B_{44}=(a c)^{4}(b c)^{4} a_{x}^{4} b_{x}^{4}$ gives $B_{44}=B_{53}+B_{431}$, i.e.,

$$
B_{44}=\frac{6}{5} B_{53}-\frac{1}{5} B_{62} \text {. }
$$

Now, by definition,

$$
\mathscr{P}_{4,4}=(a b)^{4}(b c)^{4}(c d)^{4}(d a)^{4}=\left(F, B_{44}\right)_{8}=\frac{6}{5}\left(F, B_{53}\right)_{8}-\frac{1}{5}\left(F, B_{62}\right)_{8} .
$$

The last transvectant, which is easier to compute, is given by

$$
\left(F, B_{62}\right)_{8}=\left(d_{x}^{8},(a c)^{2}(b c)^{6} a_{x}^{6} b_{x}^{2}\right)_{8}=(d a)^{6}(d b)^{2}(a c)^{2}(b c)^{6} .
$$

We now apply the Clebsch-Gordan (CG) identity [1, Eq. 2.9] to the parallel groups of two brackets each $(d b)^{2}$ and $(a c)^{2}$. This results in the formula

$$
\left(F, B_{62}\right)_{8}=\sum_{j=0}^{2} \frac{\left(\begin{array}{l}
2 \\
j
\end{array}\right)^{2}}{\left(\begin{array}{c}
5-j \\
j
\end{array}\right)}\left((F, F)_{6+j},(F, F)_{6+j}\right)_{4-2 j} .
$$

Since odd transvectants of a form with itself vanish, only the terms $j=0,2$ survive and the resulting expression readily simplifies to

$$
\left(F, B_{62}\right)_{8}=J_{4}+\frac{1}{3} J_{2}^{2} .
$$

Similarly, $\left(F, B_{53}\right)_{8}=(d a)^{5}(d b)^{3}(a c)^{3}(b c)^{5}$ to which we apply the CG identity for the bracket groups $(d b)^{3}$ and $(a c)^{3}$ with the result

$$
\left(F, B_{53}\right)_{8}=\sum_{j=0}^{3} \frac{\left(\begin{array}{c}
3 \\
j
\end{array}\right)^{2}}{\left(\begin{array}{c}
7-j \\
j
\end{array}\right)}\left((F, F)_{5+j},(F, F)_{5+j}\right)_{6-2 j}
$$


Only the $j=1,3$ terms survive and the sum simplifies to $\left(F, B_{53}\right)_{8}=\frac{3}{2} J_{4}+\frac{1}{4} J_{2}^{2}$. Hence

$$
\mathscr{P}_{4,4}=\frac{8}{5} J_{4}+\frac{7}{30} J_{2}^{2} .
$$

We now consider $\mathscr{P}_{4,5}=(a b)^{4}(b c)^{4}(c d)^{4}(d e)^{4}(e a)^{4}$ and apply the CG identity to the bracket groups $(e a)^{4}$ and $(c d)^{4}$. This gives

$$
\begin{aligned}
\mathscr{P}_{4,5} & =\sum_{j=0}^{4} \frac{\left(\begin{array}{c}
4 \\
j
\end{array}\right)^{2}}{\left(\begin{array}{c}
9-j \\
j
\end{array}\right)}\left((F, F)_{4+j},(a b)^{4}(b c)^{4}(a c)^{j} a_{x}^{4-j} c_{x}^{4-j}\right)_{8-2 j} \\
& =\left((F, F)_{4}, B_{44}\right)_{8}+\frac{12}{7}\left((F, F)_{6}, A_{22}\right)_{4}+\frac{1}{5} J_{2} J_{3} .
\end{aligned}
$$

The middle term in (9) is easy to handle thanks to (7). Indeed,

$$
\left((F, F)_{6}, A_{22}\right)_{4}=\frac{1}{2}\left((F, F)_{6}, A_{4}\right)_{4}=\frac{1}{2} J_{5} .
$$

Using (8), the computation of the first term in (9) reduces to that of $\left((F, F)_{4}, B_{53}\right)_{8}$ and $\left((F, F)_{4}, B_{62}\right)_{8}$. We have

$$
\left((F, F)_{4}, B_{62}\right)_{8}=\left((d e)^{4} d_{x}^{4} e_{x}^{4},(a c)^{2}(b c)^{6} a_{x}^{6} b_{x}^{2}\right)_{8}=\frac{2 \times 4 \times 3 \times 6 !}{8 !} C_{1}+\frac{8 \times 4 \times 6 !}{8 !} C_{2}
$$

where

$$
C_{1}=(d e)^{4}(d a)^{2}(d b)^{2}(e a)^{4}(a c)^{2}(b c)^{6} \quad, \quad C_{2}=(d e)^{4}(d a)^{3}(d b)(e b)(e a)^{3}(a c)^{2}(b c)^{6} .
$$

This formula is obtained by expanding the transvectant into bracket monomials, similarly to summing over Wick contractions in quantum field theory followed by collecting "topologically equivalent graphs". Here, this means one has to symmetrize over the matchings of the 8 factors carrying an $x$ on the left to the 8 ones on the right. The combinatorial weights can be computed "probabilistically" as follows. The two $b_{x}$ factors can either connect to the same $d$ or $e$ vertex or to each of the two vertices. The $C_{1}$ and $C_{2}$ terms above respectively correspond to these two possibilities. For the $C_{1}$ term one has 2 choices for which vertex receives the two connections. Say it is $d$. Then one has 4 choices to connect the first $b_{x}$ and 3 more choices for the second one. Finally there are 6 remaining $x$-carrying factors on the left which will all connect to the $a$ vertex and this accounts for 6 ! more choices. The numerator for the $C_{2}$ term is obtained by a similar counting argument.

By applying the CG identity to the bracket groups $(d b)^{2}$ and $(a c)^{2}$ one finds

$$
\begin{aligned}
\left((F, F)_{4}, C_{1}\right)_{8} & =\sum_{j=0}^{2} \frac{\left(\begin{array}{c}
2 \\
j
\end{array}\right)^{2}}{\left(\begin{array}{c}
5-j \\
j
\end{array}\right)}\left((d e)^{4}(e a)^{4}(d a)^{2+j} d_{x}^{2-j} a_{x}^{2-j},(b c)^{6+j} b_{x}^{2-j} c_{x}^{2-j}\right)_{4-2 j} \\
& =\left(A_{22},(F, F)_{6}\right)_{4}+\frac{1}{3} J_{3} J_{2}=\frac{1}{2} J_{5}+\frac{1}{3} J_{2} J_{3}
\end{aligned}
$$

since the $j=1$ term vanishes.

The $C_{2}$ term needs more care since we will now apply the CG identity to the pair of bracket groups $(d b)(e b)$ and $(a c)^{2}$. This gives, in redundant but hopefully self-explanatory form,

$$
C_{2}=\frac{\left(\begin{array}{c}
2 \\
0
\end{array}\right)^{2}}{\left(\begin{array}{c}
5-0 \\
0
\end{array}\right)}\left(A_{211},(F, F)_{6}\right)_{4}+\frac{\left(\begin{array}{c}
2 \\
2
\end{array}\right)^{2}}{\left(\begin{array}{c}
5-2 \\
2
\end{array}\right)}\left(J_{3},(F, F)_{8}\right)_{0}
$$


since, again the $j=1$ term is absent because $(F, F)_{7}=0$. Since we have shown that $A_{211}=0$, we get $C_{2}=\frac{1}{3} J_{2} J_{3}$ and therefore

$$
\left((F, F)_{4}, B_{62}\right)_{8}=\frac{3}{7}\left(\frac{1}{2} J_{5}+\frac{1}{3} J_{2} J_{3}\right)+\frac{4}{7}\left(\frac{1}{3} J_{2} J_{3}\right)=\frac{3}{14} J_{5}+\frac{1}{3} J_{2} J_{3} .
$$

The "probabilistic" expansion of the transvectant into bracket monomials gives

$$
\left((F, F)_{4}, B_{53}\right)_{8}=\frac{1}{7} C_{3}+\frac{6}{7} C_{4}
$$

with

$$
C_{3}=(d e)^{4}(d a)(d b)^{3}(e a)^{4}(a c)^{3}(b c)^{5} \quad, \quad C_{4}=(d e)^{4}(d a)^{2}(d b)^{2}(e a)^{3}(e b)(a c)^{3}(b c)^{5} .
$$

Applying the CG identity to $(d b)^{3}$ and $(a c)^{3}$ in $C_{1}$ gives

$$
C_{3}=\frac{\left(\begin{array}{l}
3 \\
1
\end{array}\right)^{2}}{\left(\begin{array}{l}
6 \\
1
\end{array}\right)}\left(A_{22},(F, F)_{6}\right)_{4}+\frac{\left(\begin{array}{l}
3 \\
3
\end{array}\right)^{2}}{\left(\begin{array}{l}
4 \\
3
\end{array}\right)} J_{2} J_{3}=\frac{3}{4} J_{5}+\frac{1}{4} J_{3} J_{2}
$$

Applying the CG identity to $(d b)^{2}(e b)$ and $(a c)^{3}$ in $C_{4}$, with extra care as we did for $C_{2}$, we obtain

$$
C_{4}=\frac{\left(\begin{array}{l}
3 \\
1
\end{array}\right)^{2}}{\left(\begin{array}{l}
6 \\
1
\end{array}\right)}\left(\frac{1}{3} A_{22}+\frac{2}{3} A_{211},(F, F)_{6}\right)_{4}+\frac{\left(\begin{array}{l}
3 \\
3
\end{array}\right)^{2}}{\left(\begin{array}{l}
4 \\
3
\end{array}\right)} J_{3} J_{2}=\frac{1}{4} J_{5}+\frac{1}{4} J_{2} J_{3} .
$$

Substituting back, we get

$$
\left((F, F)_{4}, B_{53}\right)_{8}=\frac{9}{28} J_{5}+\frac{1}{4} J_{2} J_{3} \quad, \quad\left((F, F)_{4}, B_{44}\right)_{8}=\frac{12}{35} J_{5}+\frac{7}{30} J_{2} J_{3},
$$

and finally

$$
\mathscr{P}_{4,5}=\frac{6}{5} J_{5}+\frac{13}{30} J_{2} J_{3}
$$

We thus explicitly showed that $J_{2}, J_{3}, J_{4}, J_{5}$ and $\mathscr{P}_{4,2}, \mathscr{P}_{4,3}, \mathscr{P}_{4,4}, \mathscr{P}_{4,5}$ are triangularly related and established the following result.

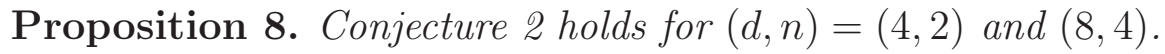

Remark 2. To the best of our knowledge, there are only two proofs of Dixmier's conjecture for the octavic. In [21, p. 137], Dixmier says that the $d=8$ case was checked by computer by Bartels and then he later also checked it "by hand". However, no details are given. The second proof [37] is also computer-assisted. Incidentally, one can use the argument of [37] to quickly derive Proposition 8 from Theorem 1. From the knowledge of the first few terms of the Hilbert series, and the algebraic independence of $\mathscr{P}_{4,2}, \mathscr{P}_{4,3}, \mathscr{P}_{4,4}, \mathscr{P}_{4,5}$ one deduces that they are triangularly related to $J_{2}, J_{3}, J_{4}, J_{5}$ and therefore constitute a regular sequence in Inv $_{8}$. Of course, this rests crucially on the prior knowledge of a HSOP with the right degrees which here is due to Shioda [42]. 


\section{Why DiXMieR's CONJECTURE IS NOT ONLY HARD BUT RIDICULOUSLY SO}

We again assume the degree of our generic binary form is $d=2 k$ with $k$ even. In order to understand the invariants $\mathscr{P}_{n, p}$ it is important to be able to see them. This is provided by the Feynman diagram calculus developed in [1], with additional explanations given in [5, $\S 4.2]$. We will not repeat here the corresponding definitions and refer the reader to these two resources. In the notations of [1, §2] one has

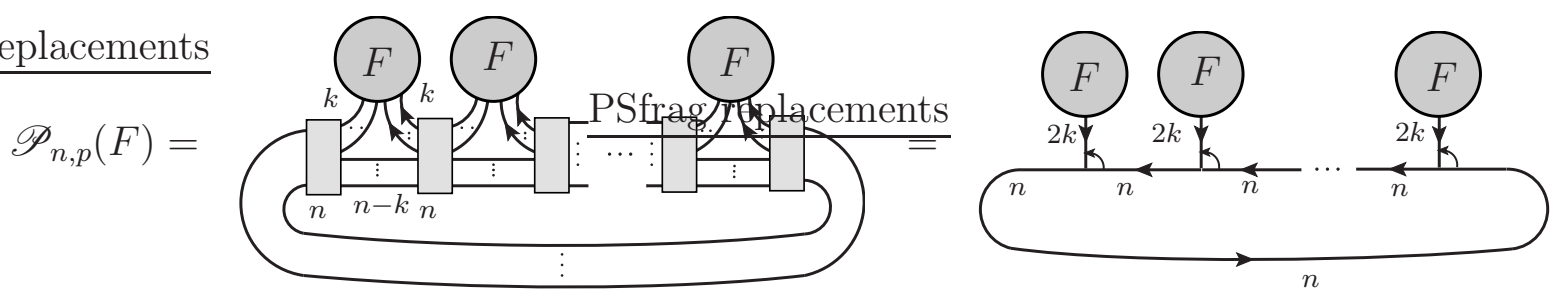

The first graphical formula uses the "microscopic" notation of [1, §2] whereas the second one uses the more compact "macroscopic" notation of the same reference. There is no numerical ambiguity (overall sign or proportionality factor) in both graphical formulas.

In the simplest quadratic case one has, for all $n \geq k$, the formula

$$
\mathscr{P}_{n, 2}(F)=\frac{(n-k) !(n+k+1) ! k !^{2}}{n !^{2}(2 k+1) !} \times(F, F)_{2 k} .
$$

The latter is an immediate consequence of the graphical form of an identity of Clebsch [1, Eq. (2.10)]. However, for the cubic invariant case one has that $\mathscr{P}_{n, 3}(F)=\alpha_{n, k} \beta_{n, k} \mathscr{P}_{k, 3}(F)$ where $\mathscr{P}_{k, 3}(F)=\left(F,(F, F)_{k}\right)_{2 k}, \alpha_{n, k}$ is a trivial nonvanishing factor ( \pm a square root of a ratio of products of factorials) and $\beta_{n, k}$ is the Wigner $6 j$ symbol

$$
\beta_{n, k}=\left\{\begin{array}{ccc}
k & k & k \\
\frac{n}{2} & \frac{n}{2} & \frac{n}{2}
\end{array}\right\}
$$

This computation is an example of star-triangle relation in the quantum theory of angular momentum. It follows from similar manipulations as in [4, Proof of Theorem 7.2]. The definition of $6 j$ symbols in relation to the invariant theory of binary forms is recalled in [3, $\S 7$. A trivial consequence of Conjecture 2 is that $\beta_{n, k}$ should not vanish. Encouraged by computer checks due to J. Van der Jeugt, we propose the following purely combinatorial conjecture.

Conjecture 3. For all pairs of integers $(k, n)$ with $n \geq k \geq 2$ and with the exception of $(k, n)=(2,3)$, one has

$$
\left\{\begin{array}{ccc}
k & k & k \\
\frac{n}{2} & \frac{n}{2} & \frac{n}{2}
\end{array}\right\} \neq 0
$$

Note that we did not assume $k$ even in the last statement. Simply by requiring the nonvanishing of the degree three invariant in Dixmier's list, it is immediate that Dixmier's conjecture would imply the particular cases $n=2 k-2$ with $k$ even and $k \geq 2$ of Conjecture 3. By Racah's formula for $6 j$ symbols [41, Appendix B] (see also [4, §7]), Conjecture 3 
amounts to the nonvanishing of the combinatorial sum

$$
\sum_{j}(-1)^{j}\left(\begin{array}{c}
j+1 \\
3 k+1
\end{array}\right)\left(\begin{array}{c}
k \\
j-k-n
\end{array}\right)^{3}
$$

for the same range of pairs $(k, n)$. A matrix plot of the Mathematica command

Table [Sign [SixJSymbol $[\{2+i, 2+i, 2+i\},\{(2+i+j) / 2,(2+i+j) / 2$,

$$
(2+i+j) / 2\}]],\{i, 0,200\},\{j, 0,200\}]
$$

produces the following picture which exhibits a rather intricate sign pattern.

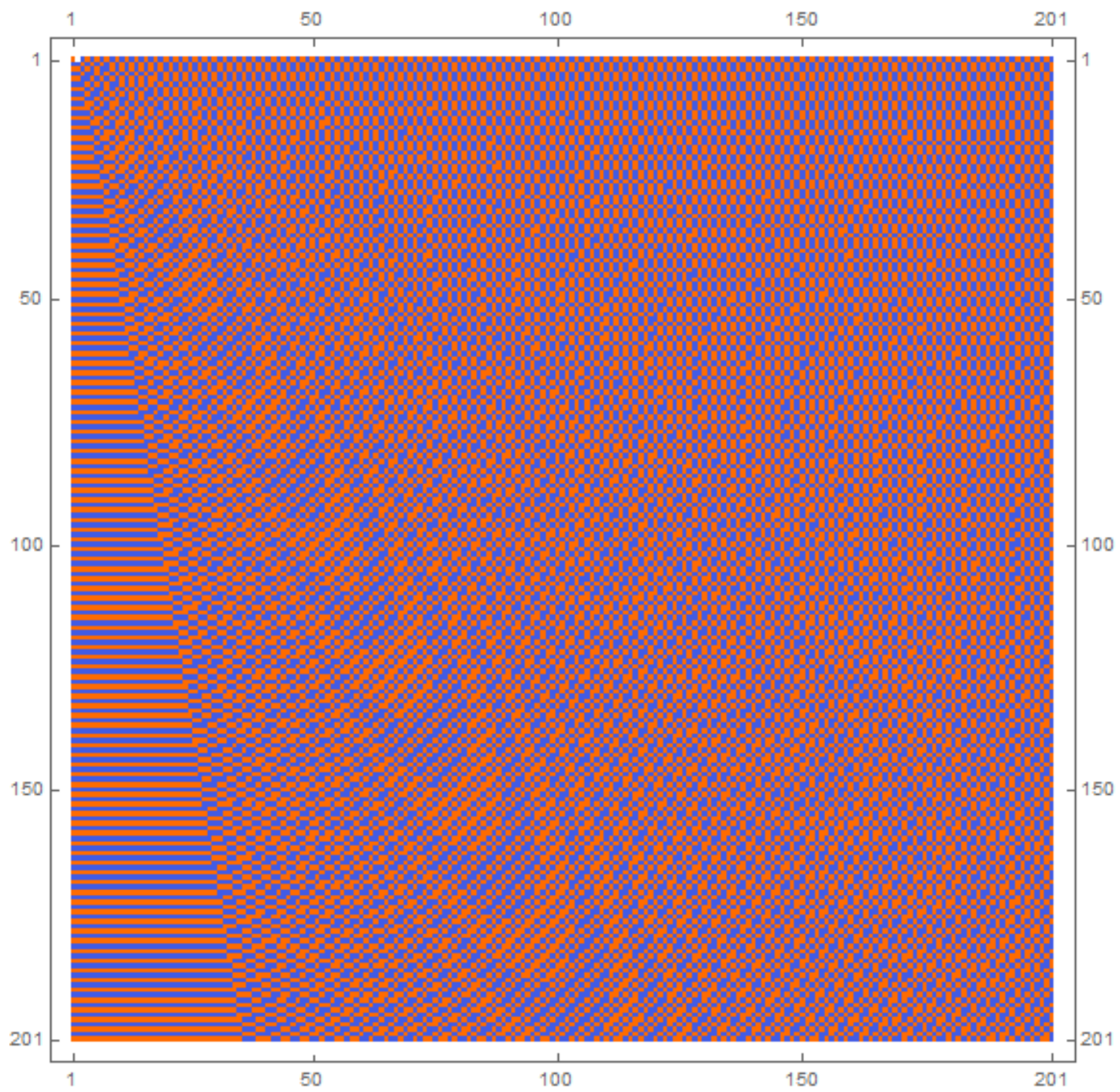

If $r=1, \ldots, 201$ is the row number from top to bottom and if $c=1, \ldots, 201$ is the column number from left to right then the coloring of the square in $r$-th row and $c$-th column of the previous picture gives the sign of the $6 j$ symbol

$$
\left\{\begin{array}{ccc}
r+1 & r+1 & r+1 \\
\frac{r+c}{2} & \frac{r+c}{2} & \frac{r+c}{2}
\end{array}\right\}
$$

Light square means positive, dark means negative and white square means zero. In general, zeros of $6 j$ symbols are poorly understood (see, e.g., [43, 45, 14]). As to the curious $(k, n)=$ 
$(2,3)$ exception or white square in the top left corner of the picture, it has been given a representation-theoretic explanation in [38]. Needless to say, the author of the present article has no idea how to prove Conjecture 3, even in the Dixmier case where $n=2 k-2$. We also learned from C. Krattenthaler that for simpler-looking combinatorial sums, similar nonvanishing conjectures are open (see, e.g., [15, Conjecture A]).

Acknowledgements: For useful discussions or correspondence the author thanks A. Brouwer, J. Chipalkatti, C. Huneke, C. Krattenthaler and J. Van der Jeugt. The author also thanks the anonymous referee for suggesting useful improvements.

Conflict of interest statement: The author states that there is no conflict of interest.

\section{REFERENCES}

[1] A. Abdesselam, On the volume conjecture for classical spin networks. J. Knot Theory Ramifications 21 (2012), no. 3, 1250022, 62 pp.

[2] A. Abdesselam and J. Chipalkatti, On the Wronskian combinants of binary forms. J. Pure Appl. Algebra 210 (2007), no. 1, 43-61.

[3] A. Abdesselam and J. Chipalkatti, The higher transvectants are redundant. Ann. Inst. Fourier (Grenoble) 59 (2009), no. 5, 1671-1713.

[4] A. Abdesselam and J. Chipalkatti, Quadratic involutions on binary forms. Preprint arXiv:1008.3117 [math.AG], extended version of article with same authors and title with reference: Michigan Math. J. 61 (2012), no. 2, 279-296.

[5] A. Abdesselam and J. Chipalkatti, On the reconstruction problem for Pascal lines. Discrete Comput. Geom. 60 (2018), no. 2, 381-405.

[6] G. E. Andrews, Problems and prospects for basic hypergeometric functions. In: Theory and Application of Special Functions (Proc. Advanced Sem., Math. Res. Center, Univ. Wisconsin, Madison, Wis., 1975), pp. 191-224. Math. Res. Center, Univ. Wisconsin, Publ. 35, Academic Press, New York, 1975.

[7] L. Bedratyuk, A complete minimal system of covariants for the binary form of degree 7. J. Symbolic Comput. 44 (2009), no. 2, 211-220.

[8] M. Brion, Invariants et covariants des groupes algébriques réductifs. Lecture notes for the 1996 Monastir summer school "Théorie des Invariants". Available at http://www-fourier.univ-grenoble-alpes.fr/ mbrion/monastirrev.pdf

[9] A. E. Brouwer and M. Popoviciu, The invariants of the binary nonic. J. Symbolic Comput. 45 (2010), no. $6,709-720$.

[10] A. E. Brouwer and M. Popoviciu, The invariants of the binary decimic. J. Symbolic Comput. 45 (2010), no. 8, 837-843.

[11] A. E. Brouwer, J. Draisma and M. Popoviciu, The degrees of a system of parameters of the ring of invariants of a binary form. Transform. Groups 20 (2015), no. 4, 953-967.

[12] N. G. de Bruijn, Asymptotic Methods in Analysis. Corrected reprint of the third edition. Dover Publications, Inc., New York, 1981.

[13] W. Bruns and J. Herzog, Cohen-Macaulay Rings. Cambridge Studies in Advanced Mathematics 39. Cambridge University Press, Cambridge, 1993.

[14] L. Cagliero and F. Szechtman, The classification of uniserial $\mathfrak{s l}(2) \ltimes V(m)$-modules and a new interpretation of the Racah-Wigner $6 j$-symbol. J. Algebra 386 (2013), 142-175.

[15] A. Carnevale and C. Voll, Orbit Dirichlet series and multiset permutations. Monatsh. Math. 186 (2018), no. 2, 215-233.

[16] J. Chipalkatti, On Hermite's invariant for binary quintics. J. Algebra 317 (2007), no. 1, 324-353.

[17] F. Cléry, C. Faber and G. van der Geer, Covariants of binary sextics and vector-valued Siegel modular forms of genus two. Math. Ann. 369 (2017), no. 3-4, 1649-1669.

[18] H. Cröni, Zur Berechnung von Kovarianten von Quantiken. Ph.D. Thesis, Univ. des Saarlandes, Saarbrücken, 2002.

[19] H. Derksen, Universal denominators of Hilbert series. J. Algebra 285 (2005), no. 2, 586-607. 
[20] H. Derksen and G. Kemper, Computational Invariant Theory. Invariant Theory and Algebraic Transformation Groups, I. Encyclopaedia of Mathematical Sciences 130. Springer-Verlag, Berlin, 2002.

[21] J. Dixmier, Quelques résultats et conjectures concernant les séries de Poincaré des invariants des formes binaires. Séminaire d'algèbre Paul Dubreil et Marie-Paule Malliavin, 36ème année (Paris, 1983-1984), pp. 127-160, Lecture Notes in Math. 1146, Springer, Berlin, 1985.

[22] J. Dixmier and D. Lazard, Le nombre minimum d'invariants fondamentaux pour les formes binaires de degré 7. Portugalia Math. 43 (1985/86), no. 3, 377-392.

[23] A. C. Dixon, On the sum of the cubes of the coefficients in a certain expansion by the binomial theorem. Messenger of Math. 20 (1890/1891), 79-80.

[24] A. C. Dixon, Summation of a certain Series. Proc. London Math. Soc. 35 (1903), 284-289.

[25] J. E. Fjeldstad, A generalization of Dixon's formula. Math. Scandinavica 2 (1954), 46-48.

[26] A. von Gall, Das vollständige Formensystem einer binären Form achter Ordnung. Math. Ann. 17 (1880), 31-51.

[27] A. von Gall, Ueber das vollständige Formensystem einer binären Form achter Ordnung. Math. Ann. 17 (1880), 139-152.

[28] A. von Gall, Auszug aus einem Brief an die Redactcion der Annalen. Math. Ann. 17 (1880), 456.

[29] A. von Gall, Das vollständige Formensystem der binären Form 7ter Ordnung. Math. Ann. 31 (1888), $318-336$.

[30] I. M. Gessel, Super ballot numbers. J. Symbolic Comput. 14 (1992), no. 2-3, 179-194.

[31] P. Gordan, Beweis, dass jede Covariante und Invariante einer binären Form eine ganze Function mit numerischen Coefficienten einer endlichen Anzahl solcher Formen ist. J. Reine Angew. Math. 69 (1868), $323-354$.

[32] V. J. W. Guo, F. Jouhet and J. Zeng, Factors of alternating sums of products of binomial and $q-$ binomial coefficients. Acta Arith. 127 (2007), no. 1, 17-31.

[33] D. Hilbert, Ueber einen allgemeinen Gesichtspunkt für invariantentheoretische Untersuchungen im binären Formengebiete, Math. Ann. 28 (1886), 381-446. For English translation see: Hilberts Invariant Theory Papers, translated from the German by M. Ackerman, with comments by R. Hermann, Lie Groups: History, Frontiers and Applications, vol. 8, Math. Sci. Press, Brookline, MA, 1978.

[34] J.-I. Igusa, Modular forms and projective invariants. American J. Math. 89 (1967), 817-855.

[35] V. G. Kac, Root systems, representations of quivers and invariant theory. In: Invariant theory (Montecatini, 1982), pp. 74-108, Lecture Notes in Math. 996, Springer, Berlin, 1983.

[36] R. Lercier and M. Olive, Covariant algebra of the binary nonic and the binary decimic. In: Arithmetic, Geometry, Cryptography and Coding Theory, pp. 65-91, Contemp. Math. 686, American Math. Soc., Providence, RI, 2017.

[37] P. Littelmann and C. Procesi, On the Poincaré series of the invariants of binary forms. J. Algebra 133 (1990), no. 2, 490-499.

[38] P. Minnaert, Construction of the superalgebra $g l(2 \mid 2)$ from $s o(3)$ tensor operators and the vanishing of the 6- $j$ symbol $\{222 \mid 3 / 23 / 23 / 2\}$. Europhys. Lett. 12 (1990), no. 2, 97-100.

[39] M. Olive, About Gordan's algorithm for binary forms. Found. Comput. Math. 17 (2017), no. 6, 14071466.

[40] V. L. Popov, Homological dimension of algebras of invariants. J. Reine Angew. Math. 341 (1983), $157-173$.

[41] G. Racah, Theory of Complex Spectra. II. Phys. Rev. 62 (1942), 438-462.

[42] T. Shioda, On the graded ring of invariants of binary octavics. American J. Math. 89 (1967), 10221046.

[43] K. Srinivasa Rao, V. Rajeswari and R. C. King, Solutions of Diophantine equations and degree-one polynomial zeros of Racah coefficients. J. Phys. A 21 (1988), no. 9, 1959-1970.

[44] K. von Szily, Über die Quadratsummen der Binomialcoefficienten. Math. und Naturw. Ber. aus Ungarn $12(1893 / 1894), 84-91$.

[45] J. Van der Jeugt, Tensor product of group representations and structural zeros of Racah coefficients. J. Math. Phys. 33 (1992), no. 7, 2417-2421.

[46] J. Weyman, Gordan ideals in the theory of binary forms. J. Algebra 161 (1993), no. 2, 370-391. 
Abdelmalek Abdesselam, Department of Mathematics, P. O. Box 400137, University of Virginia, Charlottesville, VA 22904-4137, USA

E-mail address: malek@virginia.edu 